\title{
Expression of heat-shock protein-90 in non-Hodgkin's lymphomas
}

Jose R Valbuena ${ }^{1}$, George Z Rassidakis ${ }^{1}$, Pei Lin ${ }^{1}$, Coralyn Atwell ${ }^{1}$, Georgios V Georgakis ${ }^{2}$, Anas Younes ${ }^{2}$, Dan Jones ${ }^{1}$ and L Jeffrey Medeiros ${ }^{1}$

${ }^{1}$ Department of Hematopathology, The University of Texas MD Anderson Cancer Center, Houston, TX, USA; and ${ }^{2}$ Department of Lymphoma-Myeloma, The University of Texas MD Anderson Cancer Center, Houston,

TX, USA

\begin{abstract}
Heat-shock protein-90 (HSP90) inhibitors are currently being used in phase I clinical trials for treating patients with a variety of neoplasms including lymphomas. Using immunohistochemical methods, we assessed for HSP90 expression in $\mathbf{4 1 2}$ cases of non-Hodgkin's lymphoma. In B-cell lymphomas, HSP90 was moderately to strongly expressed in all cases of Burkitt's lymphoma (5/5,100\%), and in subsets of follicular lymphoma (17/28, $61 \%)$, diffuse large B-cell lymphoma $(27 / 46,59 \%)$, nodal marginal zone B-cell lymphoma $(6 / 16,38 \%)$, plasma cell neoplasms $(14 / 39,36 \%)$, small lymphocytic lymphoma/chronic lymphocytic leukemia $(3 / 9,33 \%)$, mantle cell lymphoma $(12 / 38,32 \%)$ and lymphoplasmacytic lymphoma/Waldenstrom macroglobulinemia $(3 / 10,30 \%)$. HSP90 was weakly expressed in six of $14(43 \%)$ cases of extranodal marginal zone B-cell lymphoma of mucosaassociated lymphoid tissue. In T-cell lymphomas, HSP90 was moderately to strongly expressed in subsets of anaplastic large-cell lymphoma (14/24, 58\%; 9/12 ALK + and 5/12 ALK-), precursor-T-cell lymphoblastic leukemia/lymphoma $(20 / 65,31 \%)$, unspecified peripheral T-cell lymphoma $(8 / 43,23 \%)$ and angioimmunoblastic T-cell lymphoma $(2 / 17,12 \%)$. HSP90 was weakly expressed in seven of $58(12 \%)$ cases of mycosis fungoides. We conclude that HSP90 is commonly expressed in a subset of many types of B- and T-cell lymphoma. These data suggest that many lymphoma types are suitable targets for modulation of HSP90 activity, and that HSP90 inhibitors are a potential investigational therapy for lymphoma patients.
\end{abstract}

Modern Pathology (2005) 18, 1343-1349. doi:10.1038/modpathol.3800459; published online 15 July 2005

Keywords: HSP90; NHL; immunohistochemistry

Heat-shock proteins (HSPs), so named because they were initially observed in cells exposed to elevated temperature, are molecular chaperones. ${ }^{1,2}$ Molecular chaperones are cytosolic molecules that assist in the folding of nascent polypeptides into functional tertiary structures as they exit the ribosome, thus avoiding misfolding and aggregation in the crowded intracellular compartments., ${ }^{1,2}$ Molecular chaperones are also important in regulating the balance between protein synthesis and degradation. ${ }^{1,2}$

HSPs have been identified in diverse organisms, ranging from bacteria to humans, and are classified on the basis of their molecular weight. ${ }^{3,4}$ The four major groups include HSP110, HSP90, HSP70 and HSP27. In addition to their function in folding nascent polypeptides, HSPs are differentially ex-

Correspondence: Dr LJ Medeiros, MD, Department of Hematopathology, The University of Texas MD Anderson Cancer Center, 1515 Holcombe Boulevard, Box 72, Houston, TX 77030, USA.

E-mail: jmedeiro@mdanderson.org

Received 1 June 2005; revised and accepted 7 June 2005; published online 15 July 2005 pressed in response to cellular stress. ${ }^{3-5}$ Numerous client proteins of varied functions interact with HSPs, ranging from kinases (both tyrosine and serine/threonine kinases) to transcription factors to proteins involved in cell cycle regulation. ${ }^{5}$ HSPs play a pivotal role in protecting cells from apoptosis, thereby prolonging survival. ${ }^{3-7}$

In recent years, the molecular chaperone that has emerged as the most promising target of innovative cancer therapy is HSP90. HSP90 is ubiquitously expressed in the cytoplasm of normal cells where it binds with many client proteins and is involved in homeostasis. ${ }^{1-7}$ In normal cells, HSP90 is expressed at relatively low levels and does not form complexes with other chaperone proteins. ${ }^{8}$ Thus, others have suggested that HSP90 is present in latent form in normal cells. By contrast, in neoplastic cells HSP90 is expressed at much higher levels, two to 10 times greater, suggesting that HSP90 provides a survival advantage for neoplastic cells. HSP90 also forms large complexes with other chaperone proteins in neoplastic cells to form the so-called multichaperone or superchaperone complexes. ${ }^{8,9}$ 
HSP90 has three domains: a C-terminal domain, a middle domain and an $\mathrm{N}$-terminal domain. The $\mathrm{C}$ terminal domain is involved in homodimerization and docking with other cochaperone proteins. The middle domain contributes to client protein binding and the N-terminal domain is most important, as this is the site of an ATP-binding pocket that is essential for client protein binding. ${ }^{9}$ Via the N-terminal domain, HSP90 serves as a molecular chaperone for many client proteins that are involved in oncogenesis, for example, NF-kappa B, BCR-ABL, NPM-ALK, AKT and mutated p53. ${ }^{8-20}$ As these client proteins are involved in a number of pathways that are critical to the survival of neoplastic cells, HSP90 is an attractive target for inhibition because many pathways can be blocked simultaneously. It therefore follows that HSP90 inhibitors could be active against many tumor types.

Inhibitors of HSP90 have been developed, and these act by competing with ATP for binding to the $\mathrm{N}$-terminal domain of HSP90. The first inhibitor suitable for clinical trials, 17-allylamino-17-desmethoxygeldanamycin (17-AAG), was shown in preclinical studies to induce apoptosis in cell lines derived from solid and hematologic neoplasms. ${ }^{9} \mathrm{~A}$ number of phase I clinical trials using 17-AAG, either alone or in combination with other agents, are currently underway. ${ }^{9}$

At our institution, there was interest in using 17-AAG to treat patients with relapsed or refractory non-Hodgkin's lymphoma (NHL). In our search of the literature, it became clear that HSP90 expression has not been systematically assessed in most types of NHL, prompting this study. Thus, we have used immunohistochemical methods and Western blot analysis to assess for HSP90 expression in a variety of types of B- and T-cell NHL and in cell lines, classified using the World Health Organization classification system.

\section{Materials and methods}

\section{Case Selection}

A total of 412 cases of NHL were collected from the files of the Department of Hematopathology, The University of Texas MD Anderson Cancer Center, between 1996 and 2004. All cases were diagnosed and classified using morphologic and immunophenotypic criteria specified in the World Health Organization Classification of lymphoid neoplasms. ${ }^{21}$

The group of B-cell NHL cases assessed included: 46 diffuse large B-cell lymphoma (including eight of the immunoblastic variant), 39 plasma cell neoplasms (34 myeloma, five plasmacytoma), 38 mantle cell lymphoma (26 small cell or typical and 12 blastoid), 28 follicular lymphoma (eight grade 1, 11 grade 2, nine grade 3 ), 16 nodal marginal zone B-cell lymphoma, 14 extranodal marginal zone
B-cell lymphoma of mucosa-associated lymphoid tissue (also known as MALT-lymphoma), 10 lymphoplasmacytic lymphoma/Waldenstrom macroglobulinemia, nine small lymphocytic lymphoma/ chronic lymphocytic leukemia and five Burkitt's lymphoma.

The group of T-cell NHL cases assessed included: 65 precursor T-cell lymphoblastic leukemia/lymphoma, 58 mycosis fungoides, 43 unspecified peripheral T-cell lymphoma, 24 anaplastic large-cell lymphoma (12 ALK +, 12 ALK-) and 17 angioimmunoblastic T-cell lymphoma. We also analyzed eight reactive lymph node biopsy specimens. These specimens included five cases of follicular hyperplasia, one case of follicular hyperplasia with progressive transformation of germinal centers, one case of monocytoid B-cell hyperplasia and one case with a paracortical immunoblastic proliferation.

All cases were analyzed using either tissue microarrays $(n=325)$ or full tissue sections $(n=87)$. The tissue microarrays included duplicate, triplicate or quadruplet tumor cores from all cases and were constructed using a manual tissue arrayer (Beecher Instruments, Silver Springs, MD, USA) as described previously. ${ }^{22}$ All neoplasms assessed were fixed in $10 \%$ buffered formalin, routinely processed and embedded in paraffin.

\section{Cell lines, Protein Extraction and Western Blot Analysis}

In all, 12 lymphoma cell lines were evaluated by Western blot analysis, including three mantle cell lymphoma (Mino, SP-53 and JM-P1), two diffuse large B-cell lymphoma (MS and JM), two ALK + anaplastic large-cell lymphoma (Karpas 299 and SUDHL-1), one primary effusion lymphoma (BC-1), one multiple myeloma (IM-9), one Burkitt's lymphoma (Daudi), one ALK - cutaneous anaplastic large-cell lymphoma (Mac2a) and one precursor T-cell lymphoblastic leukemia/lymphoma (Jurkat). Cell lines were maintained in RPMI 1640 medium supplemented with $1 \%$ nonessential amino acids, $10 \%$ fetal calf serum (Life Technologies Inc., Rockville, MD, USA), and $1 \%$ streptomycin-penicillin. Cell cultures were incubated at $37^{\circ} \mathrm{C}$ in a humidified atmosphere containing $5 \% \mathrm{CO}_{2}$.

Cell lysates ( $50 \mu \mathrm{g}$ protein) were prepared for Western blot analysis as decribed previously. ${ }^{23} \mathrm{~A}$ monoclonal antibody specific for HSP90 (1:200 dilution, Novocastra Laboratories, Newcastle-uponTyne, UK) was used. Reactions were visualized using a secondary antibody conjugated with horseradish peroxidase (BioRad, Hercules, CA, USA) and enhanced chemiluminescence reagents (Amersham Pharmacia, Piscataway, NJ, USA). $\beta$ actin (1:2000 dilution, Sigma, St Louis, MO, USA) served as a positive control for protein load and integrity. 


\section{Immunohistochemical Methods}

The immunohistochemical methods used in this study have been described previously. ${ }^{23}$ Heatinduced antigen retrieval was performed prior to immunohistochemical staining. Sections were incubated with a HSP90 monoclonal antibody (1:200 dilution, Novocastra Laboratories) at $4^{\circ} \mathrm{C}$ overnight. Detection of signal was achieved using the LSAB + kit (DAKO, Carpinteria, CA, USA) that contains secondary biotinylated antibody and streptavidin/horseradish peroxidase complex, according to the manufacturer's recommendations. $3,3^{\prime}$-diaminobenzidine $/ \mathrm{H}_{2} \mathrm{O}_{2}$ (DAKO) was used as chromogen and slides were counterstained with hematoxylin.

\section{Scoring of Positive Cells}

An arbitrary $20 \%$ cutoff was used to determine HSP90 positivity. However, in most positive cases, the percentage of HSP90-positive cells was more than $50 \%$ of tumor cells. Only cytoplasmic staining was considered positive for HSP90, and expression was graded visually as negative, weak or moderate/ strong. A case was considered weakly positive if cytoplasmic staining was similar to or less than that of normal small $\mathrm{T}$ cells, and moderate to strongly positive if cytoplasmic staining was similar to or greater than that of normal centroblasts and/or immunoblasts. Nuclear staining of HSP90 was seen in a small subset of cases of plasma cell myeloma and mantle cell lymphoma. However, nuclear staining was not considered positive for the purposes of this study because HSP90 exerts most of its known biological functions in the cytoplasm. The explanation for the nuclear staining in this small subset of cases is unknown; artifact cannot be excluded.

\section{Results}

\section{Cell Lines}

Western blot analysis showed that HSP90 was detected as a $90 \mathrm{kD}$ protein in all cell lines (Figure 1). HSP90 was strongly expressed in two mantle cell lymphoma cell lines (Mino and SP-53), two diffuse large B-cell lymphoma cell lines (MS and JM), one ALK + anaplastic large-cell lymphoma cell line (Karpas 299) and the ALK - cutaneous anaplastic large-cell lymphoma (Mac2a), plasma cell myeloma (IM-9) and Burkitt's lymphoma (Daudi) cell lines. Weaker HSP90 expression was detected in one mantle cell lymphoma cell line (JM-P1), one ALK+ anaplastic large-cell lymphoma cell line (SUDHL-1), and the primary effusion lymphoma (BC-1) and precursor T-cell lymphoblastic leukemia/lymphoma (Jurkat) cell lines.

\section{Reactive Lymphoid Tissues}

In all reactive lymph nodes, HSP90 was moderately to strongly expressed in germinal center cells of follicles, with the greatest intensity in centroblasts. HSP90 expression in centrocytes was moderate. Most tingible bodies macrophages and follicular dendritic cells also demonstrated strong cytoplasmic HSP90 expression (Figure 2a). In the paracortical areas, weak HSP90 expression was observed in the cytoplasm of small T cells (range, approximately 10-30\%) and in scattered histiocytes. Variable expression was observed in plasma cells, approximately half of the plasma cells were positive, ranging from weak to moderate/strong expression. In the case with monocytoid B cell hyperplasia, moderate HSP90 expression was also observed in monocytoid B cells. In the case with a florid paracortical immunoblastic proliferation, B- and T-cell immunoblasts showed moderate-to-strong HSP90 expression.

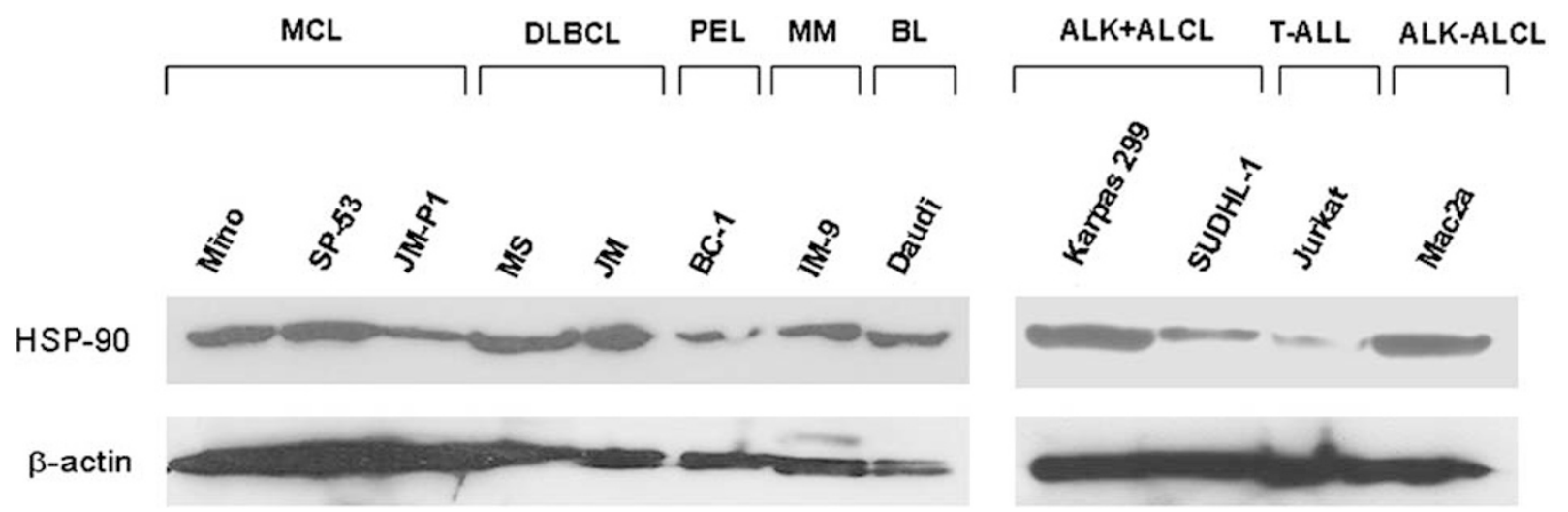

Figure 1 Western blot analysis of cell lines. The specificity of the HSP90 antibody was confirmed by detection of a $90 \mathrm{kDa}$ protein. Strong HSP90 expression was detected in two mantle cell lymphoma cell lines (Mino and SP-53), two diffuse large B-cell lymphoma cell lines (MS and JM), one ALK + anaplastic large-cell lymphoma cell line (Karpas 299), one multiple myeloma cell line (IM-9), one Burkitt's lymphoma cell line (Daudi) and one ALK - cutaneous anaplastic large-cell lymphoma cell line (Mac2a). Weak or moderate intensity of expression was observed in one mantle cell lymphoma cell line (JM-P1), one ALK + anaplastic large-cell lymphoma cell line (SUDHL-1) and the primary effusion lymphoma (BC-1) and precursor T-cell lymphoblastic leukemia/lymphoma (Jurkat) cell lines. Detection of $\beta$-actin expression served as an internal control. 

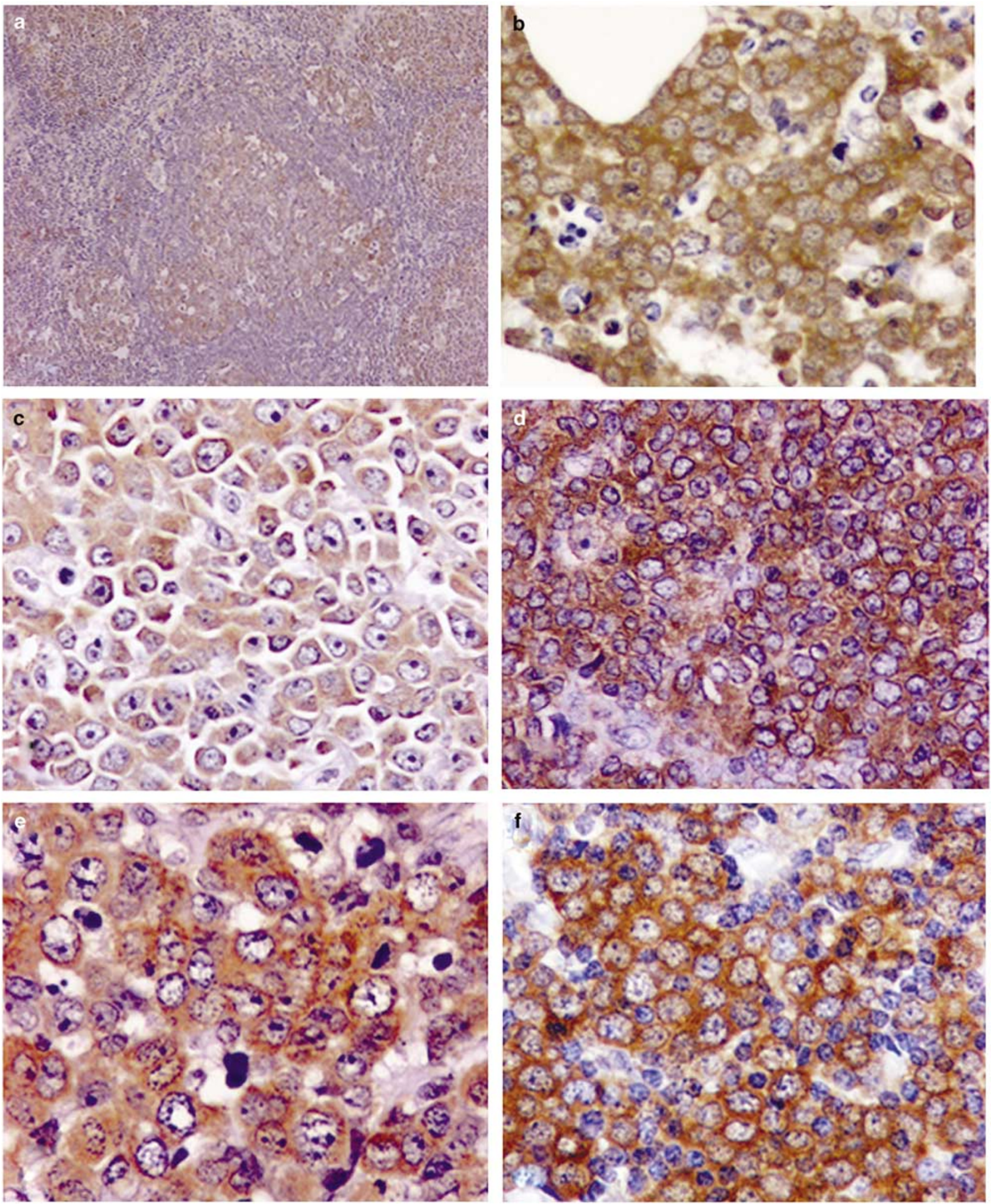

Figure 2 HSP90 expression in reactive lymphoid tissue and B-cell lymphomas. (a) In reactive lymphoid tissue, moderate staining was observed within the germinal centers, and weak staining was observed in a subset of small reactive $\mathrm{T}$ cells in the paracortical areas. (b) Burkitt's lymphoma with diffuse and strong staining for HSP90. (c-f) Moderate-to-strong expression of HSP90 was also observed in a subsets of diffuse large B-cell lymphoma (c), mantle cell lymphoma with blastoid morphology (d), ALK + anaplastic largecell lymphoma (e) and precursor T-cell lymphoblastic leukemia/lymphoma (f). Immunohistochemistry with hematoxylin counterstain (a, × 200; b-f, $\times 500)$. 


\section{Non-Hodgkin's Lymphomas}

The results of HSP90 immunostaining in various types of NHL are summarized in Table 1.

The only type of NHL in which HSP90 was invariably and strongly expressed was Burkitt's lymphoma, in all five cases assessed (Figure 2b). In other tumor types, subsets of tumors were moderately/strongly positive, weakly positive or negative. Of greatest interest are the moderate-tostrongly positive neoplasms as these tumors are most likely to be susceptible to HSP inhibitors. Thus, these cases are specifically discussed in more detail here. The physiologic significance of weakly expressed HSP90 is uncertain, but it seems likely that pharmacologic inhibition of HSP90 in these neoplasms is less likely.

Moderate-to-strong HSP90 expression was detected in 17 of $28(61 \%)$ cases of follicular lymphoma, and was restricted to grade $2(n=9)$ and grade $3(n=8)$ neoplasms. In diffuse large B-cell lymphoma, HSP90 was moderate to strongly expressed in 27 of 46 (59\%) cases (Figure 2c). All eight cases $(100 \%)$ of the immunoblastic variant were

Table 1 Summary of HSP90 expression in NHLs

\begin{tabular}{|c|c|c|c|c|}
\hline \multirow[t]{2}{*}{ Lymphoma type } & \multicolumn{2}{|c|}{ HSP9O } & \multicolumn{2}{|c|}{ Expression } \\
\hline & $\begin{array}{l}\text { Total } \\
\text { cases }\end{array}$ & $\begin{array}{c}\text { Negative, } \\
\mathrm{N}(\%)\end{array}$ & $\begin{array}{l}\text { Weak, } \\
\text { N (\%) }\end{array}$ & $\begin{array}{c}\text { Moderate/ } \\
\text { strong, N (\%) }\end{array}$ \\
\hline$N H L$ & 412 & & & \\
\hline$F L$ & 28 & 8 (29) & $3(11)$ & $17(61)$ \\
\hline Grade 1 & 8 & 6 & 2 & 0 \\
\hline Grade 2 & 11 & 1 & 1 & 9 \\
\hline Grade 3 & 9 & 1 & 0 & 8 \\
\hline B-SLL/CLL & 9 & $2(22)$ & $4(45)$ & $3(33)$ \\
\hline ENMZL & 14 & $8(57)$ & $6(43)$ & $0(0)$ \\
\hline NMZL & 16 & $2(13)$ & $8(50)$ & $6(38)$ \\
\hline MCL & 38 & $20(53)$ & $6(15)$ & $12(32)$ \\
\hline Typical & 26 & 17 & 3 & 6 \\
\hline Blastoid & 12 & 3 & 3 & 6 \\
\hline LPL/WM & 10 & $4(40)$ & $3(30)$ & $3(30)$ \\
\hline PCN & 39 & $15(38)$ & $10(26)$ & $14(36)$ \\
\hline DLBCL & 46 & $10(24)$ & $9(21)$ & $27(59)$ \\
\hline $\mathrm{BL}$ & 5 & $0(0)$ & $0(0)$ & $5(100)$ \\
\hline ALK+ ALCL & 12 & $0(0)$ & $3(25)$ & $2(50)$ \\
\hline ALK - ALCL & 12 & $5(42)$ & $2(16)$ & $5(42)$ \\
\hline AITL & 17 & $2(12)$ & $13(76)$ & $2(12)$ \\
\hline T-ALL/LBL & 65 & $23(35)$ & $22(34)$ & $20(31)$ \\
\hline PTCL, U & 43 & $17(40)$ & $16(37)$ & $8(23)$ \\
\hline $\mathrm{MF}$ & 58 & $51(88)$ & 7 (12) & $0(0)$ \\
\hline
\end{tabular}

NHL = non-Hodgkin's lymphoma; $N=$ number of cases; $F L=$ follicular lymphoma; SLL/CLL = small lymphocytic lymphoma/chronic lymphocytic leukemia; ENMZL = extranodal marginal zone B-cell lymphoma; MCL = mantle cell lymphoma; LPL/WM = lymphoplasmacytic lymphoma/Waldenstrom macroglobulemia; $\mathrm{PCN}=$ plasma cell neoplasms; NMZL = nodal marginal zone B-cell lymphoma; DLBCL = diffuse large B-cell lymphoma; BL = Burkitt's lymphoma; ALCL $=$ anaplastic large-cell lymphoma; AITL $=$ angioimmunoblastic T-cell lymphoma; ALL/LBL = acute lymphoblastic leukemia/lymphoma; PTCL; $\mathrm{U}=$ peripheral T-cell lymphoma, unspecified; $\mathrm{MF}=$ mycosis fungoides. positive, compared with 19 of 38 (50\%) centroblastic neoplasms. Four cases of diffuse large B-cell lymphoma arising in patients with small lymphocytic lymphoma/chronic lymphocytic leukemia (socalled Richter's syndrome) were studied; all strongly positive for HSP90.

Other types of B-cell NHL moderately or strongly expressed HSP90 in $30-40 \%$ of cases. HSP90 was expressed in 14 of $39(36 \%)$ plasma cell neoplasms, including two of five plasmacytoma, and 12 of 34 plasma cell myeloma. There were no qualitative or quantitative differences in staining between plasmacytoma and plasma cell myeloma. HSP90 was expressed in six of 16 (38\%) nodal marginal zone Bcell lymphomas. Intensity of expression was greatest in areas that exhibited more prominent monocytoid differentiation. Three of nine $(33 \%)$ cases of small lymphocytic lymphoma/chronic lymphocytic leukemia were positive for HSP90. Moderate to strong expression was restricted to the proliferation centers. The remainder of the neoplastic small lymphocytes were weakly positive or negative. HSP90 was expressed in 12 of $38(32 \%)$ cases of mantle cell lymphoma, including six of 26 small cell and six of 12 blastoid variant (Figure 2d). In lymphoplasmacytic lymphoma/Waldestrom macroglobulinemia, moderate-to-strong HSP90 expression was detected in three of 10 cases (30\%), and correlated with areas of more prominent plasmacytic differentiation.

In T-cell NHL, HSP90 was expressed in nine of 12 $(75 \%)$ cases of ALK + anaplastic large-cell lymphoma (Figure 2e) and five of 12 cases (42\%) of ALKanaplastic large-cell lymphoma. In precursor T-cell lymphoblastic leukemia/lymphoma, HSP90 was expressed in 20 of $65(31 \%)$ cases (Figure 2f). HSP90 was also expressed in eight of 43 (23\%) unspecified peripheral T-cell lymphoma and two of $17(12 \%)$ angioimmunoblastic T-cell lymphoma.

Two types of NHL showed only weak intensity expression of HSP90, in six of $14(43 \%)$ cases of MALT-lymphoma and seven of $58(12 \%)$ cases of mycosis fungoides. As shown in Table 1, weak HSP90 expression was also detected in a small subset of cases in most types of B- and T-cell NHL.

\section{Discussion}

The role of HSP90 in tumorigenesis has been studied in vitro in solid tumors, lymphomas and leukemias. ${ }^{8-20}$ Increasing evidence has shown that specific inhibition of HSP90 in neoplastic cells can lead to cell cycle arrest and apoptosis. ${ }^{8-20}$ No previous study, however, has systematically surveyed HSP90 expression in a variety of NHL types as defined in the WHO classification. Thus, the aim of this study was to assess for HSP90 expression in various types of B- and T-NHL, in light of the potential utility of HSP90 inhibitors in the treatment of patients with NHL. 
HSP90 was moderately to strongly expressed in a subset of many NHL types, and therefore these neoplasms are likely to be susceptible to HSP90 inhibition (Table 1). Among B-cell NHL, HSP90 was expressed in all cases of Burkitt's lymphoma $(100 \%)$. HSP90 was also expressed in $61 \%$ of follicular lymphomas, mainly grade $2(n=9)$ and grade $3(n=8)$ tumors, and $59 \%$ of diffuse large Bcell lymphoma. In the latter groups, all eight neoplasms with immunoblastic cytologic features and all four neoplasms that arose in patients were small lymphocytic lymphoma/chronic lymphocytic leukemia (Richter's syndrome) were positive. HSP90 was also moderately to strongly expressed in a subset of mantle cell lymphoma cases, and expression correlated with cytologic features, as $50 \%$ of blastoid neoplasms were positive compared with approximately $20 \%$ of small cell or typical neoplasms. Other types of B-cell NHL were positive in $30-40 \%$ of cases. In T-cell NHL, HSP90 expression was moderately to strongly expressed in ALK + and ALK- anaplastic large-cell lymphoma (75 and $42 \%$, respectively). HSP90 was also moderately to strongly expressed in a subset of cases of precursor T-cell lymphoblastic leukemia/lymphoma (31\%), unspecified peripheral T-cell lymphoma $(23 \%)$, and angioimmunoblastic T-cell lymphoma (12\%).

Taken together, these data argue that HSP90 expression is upregulated in high-grade or large-cell NHL types. The data also suggest that histologic transformation of lymphomas results in HSP90 upregulation, as HSP90 was strongly expressed in half of blastoid variant mantle cell lymphomas and in the large B-cell lymphoma component of four cases of Richter's syndrome. It is tempting to speculate that overexpression of HSP90, particularly in high-grade tumors, may play a role in tumor cell survival through interaction of HSP90 with several client proteins involved in cell proliferation and survival. In this study group, HSP90 expression also correlated with the extent of monocytoid cytologic features (in nodal marginal zone B-cell lymphoma) and with plasmacytic differentiation (in lymphoplasmacytic lymphoma/Waldenstrom macroglobulinemia).

Recent studies using gene expression profiling also have shown HSP90 expression in diffuse large B-cell lymphomas and mantle cell lymphoma, in accord with our results. Monti and colleagues ${ }^{24}$ stratified diffuse large B-cell lymphomas into three groups on the basis of their patterns of gene expression. One group, with overexpression of genes involved in B-cell differentiation or proliferation, overexpressed HSP90. De Vos and colleagues ${ }^{25}$ compared the gene expression profiles of typical and blastoid variant mantle cell lymphoma, and showed higher levels of HSP90 expression in blastoid neoplasms. Using other methods, HSP90 also has been shown to be strongly expressed in anaplastic large-cell lymphomas, in accord with our data. ${ }^{14}$

Recently, in vitro studies have shown that treatment of solid tumors and hematological malignan- cies with a naturally occurring antibiotic, geldanamycin, and its derivative, 17-AAG, inhibits HSP90 activity through blocking ATP-binding sites of the HSP90-partner complex, with subsequent proteosomal degradation and/or dephosphorylation of client proteins. ${ }^{10-20}$ These HSP inhibitors act mainly by occupying the binding site for the client protein within the HSP90-multichaperone complex and, by blocking this step, these derivatives enhance protein destabilization with subsequent shortening of protein half-life and eventual proteosomal degradation. ${ }^{9}$ Based on the promising results of preclinical studies, ${ }^{10-20}$ several phase I clinical trials with 17-AAG have been initiated in the United States and United Kingdom for patients with hematological malignancies, and solid tumors including breast, prostate and kidney cancers. ${ }^{9,26}$ With the emergence of this approach for treating patients with hematological malignancies, we were interested in assessing HSP90 expression in various NHL types.

It is of interest that HSP90 is differentially expressed in most types of NHL. As we observed the strongest pattern of expression in high-grade types of NHL, particularly Burkitt's lymphoma, ALK + anaplastic large-cell lymphoma, and diffuse large B-cell lymphoma, it seems intuitive that patients with these NHL types are most likely to benefit from HSP90 inhibitor therapy. A subset of tumors of many NHL types, however, are negative or weakly positive for HSP90 as shown by our immunohistochemical methods, suggesting that pharmacologic inhibition of HSP90 will have less value in treating patients with these neoplasms. This appears to be particularly true for patients with MALT-lymphoma, angioimmunoblastic T-cell lymphoma and mycosis fungoides.

In conclusion, HSP90 is frequently expressed in many types of NHL, including both low- and highgrade tumors, but more commonly and strongly in higher-grade tumors. Weak expression in NHL may represent an inactive form of HSP90 without biological importance. However, moderate-to-strong HSP90 expression is likely to be involved in dysregulation of several biochemical pathways related to proliferation, cell survival and apoptosis through the interaction of HSP90 with different client proteins that are known to play pivotal roles in these pathways. Our results suggest that selective inhibition of HSP90 may be a novel target for investigational therapy in subsets of patients with NHL. Immunohistochemical assessment for HSP90 also appears to be convenient method for identifying NHL patients who potentially will be most likely to benefit from HSP90 inhibitor therapy.

\section{References}

1 Young JC, Agashe VR, Siegers K, et al. Pathways of chaperone-mediated protein folding in the cytosol. Nat Rev Mol Cell Biol 2004;5:781-791. 
2 Hartl FU, Hayer-Hartl M. Molecular chaperones in the cytosol: from nascent chain to folded protein. Science 2002;295:1852-1858.

3 Maloney A, Workman P. HSP90 as a new therapeutic target for cancer therapy: the story unfolds. Expert Opin Biol Ther 2002;2:3-24.

4 Blake MJ, Buckley AR, Zhang M, et al. A novel heat shock response in prolactin-dependent $\mathrm{Nb} 2$ node lymphoma cells. J Biol Chem 1995;270:29614-29620.

5 Burrows F, Zhang H, Kamal A. Hsp90 activation and cell cycle regulation. Cell Cycle 2004;3:1530-1536.

6 Takayama S, Reed JC, Homma S. Heat-shock proteins as regulators of apoptosis. Oncogene 2003;22:9041-9047.

7 Sreedhar AS, Nardai G, Csermely P. Enhancement of complement-induced cell lysis: a novel mechanism for the anticancer effects of HSP90 inhibitors. Immunol Lett 2004;92:157-161.

8 Kamal A, Thao L, Sensintaffar J, et al. A high-affinity conformation of Hsp90 confers tumour selectivity on Hsp90 inhibitors. Nature 2003;25:407-410.

9 Isaacs JS, Xu W, Neckers L. Heat shock protein 90 as a molecular target for cancer therapeutics. Cancer Cell 2003;3:213-217.

10 Fujiwara H, Yamakuni T, Ueno M, et al. IC101 induces apoptosis by Akt dephosphorylation via an inhibition of heat shock protein 90-ATP binding activity accompanied by preventing the interaction with Akt in L1210 cells. J Pharmacol Exp Ther 2004;310:12881295.

11 Broemer M, Krappmann D, Scheidereit C. Requirement of Hsp90 for IkB kinase (IKK) biosynthesis and for constitutive and inducible IKK and NF-kB activation. Oncogene 2004;23:5378-5386.

12 Pittet JF, Lee H, Pespeni M, et al. Stress-induced inhibition of the NF-kappa B signaling pathway results from the insolubilization of the I kappa B kinase complex following its dissociation from heat shock protein 90. J Immunol 2005;174:384-394.

13 Mizuno K, Shirogane T, Shinohara A, et al. Regulation of Pim-1 by Hsp90. Biochem Biophys Res Commun 2001;281:663-669.

14 Bonvini P, Gastaldi T, Falini B, et al. Nucleophosminanaplastic lymphoma kinase (NPM-ALK), a novel Hsp90-client tyrosine kinase: down-regulation of NPM-ALK expression and tyrosine phosphorylation in $\mathrm{ALK}(+)$ CD30(+) lymphoma cells by the Hsp90 antagonist 17-allylamino,17-demethoxygeldanamycin. Cancer Res 2002;62:1559-1566.

15 Bonvini P, Dalla Rosa H, Vignes N, et al. Ubiquitination and proteasomal degradation of nucleophosmin-anaplastic lymphoma kinase induced by 17-allylamino- demethoxygeldanamycin: role of the co-chaperone carboxyl heat shock protein 70-interacting protein. Cancer Res 2004;64:3256-3264.

16 Yao Q, Nishiuchi R, Li Q, et al. FLT3 expressing leukemias are selectively sensitive to inhibitors of the molecular chaperone heat shock protein 90 through destabilization of signal transduction-associated kinases. Clin Cancer Res 2003;9:4483-4493.

17 Beliakoff J, Bagatell R, Paine-Murrieta G, et al. Hormone-refractory breast cancer remains sensitive to the antitumor activity of heat shock protein 90 inhibitors. Clin Cancer Res 2003;9:4961-4971.

$18 \mathrm{Xu} \mathrm{W,} \mathrm{Yuan} \mathrm{X,} \mathrm{Jung} \mathrm{YJ,} \mathrm{et} \mathrm{al.} \mathrm{The} \mathrm{heat} \mathrm{shock} \mathrm{protein} 90$ inhibitor geldanamycin and the ErbB inhibitor ZD1839 promote rapid PP1 phosphatase-dependent inactivation of $\mathrm{AKT}$ in ErbB2 overexpressing breast cancer cells. Cancer Res 2003;63:7777-7784.

19 Chung YL, Troy H, Banerji U, et al. Magnetic resonance spectroscopic pharmacodynamic markers of the heat shock protein 90 inhibitor 17-allylamino, 17-demethoxydeldanamycin (17AAG) in human colon cancer models. J Natl Cancer Inst 2003;95:1624-1633.

20 Guo F, Sigua C, Bali P, et al. Mechanistic role of heat shock protein 70 in Bcr-abl-mediated resistance to apoptosis in human acute leukemia cells. Blood 2005;105:1246-1255.

21 Jaffe ES, Harris NL, Stein H, Vardiman JW (eds). World Health Organization Classification of tumours. Pathology and genetics of tumours of hematopoietic and lymphoid tissues. IARC Press: Lyon, France, 2001, pp 121-235.

22 Rassidakis GZ, Jones D, Thomaides A, et al. Apoptotic rate in peripheral T-cell lymphomas. A study using a tissue microarray with validation on full tissue sections. Am J Clin Pathol 2002;118:328-334.

23 Admirand JH, Rassidakis GZ, Abruzzo LV, et al. Immunohistochemical detection of ZAP-70 in 341 cases of non-Hodgkin and Hodgkin lymphoma. Mod Pathol 2004;17:954-961.

24 Monti S, Savage KJ, Kutok JL, et al. Molecular profiling of diffuse large B-cell lymphoma identifies robust subtypes including one characterized by host inflammatory response. Blood 2005;105:1851-1861.

25 de Vos S, Krug U, Hofmann WK, et al. Cell cycle alterations in the blastoid variant of mantle cell lymphoma (MCL-BV) as detected by gene expression profiling of mantle cell lymphoma (MCL) and MCL-BV. Diagn Mol Pathol 2003;12:35-43.

26 Sausville EA, Tomaszewski JE, Ivy P. Clinical development of 17-allylamino, 17-demethoxygeldanamycin. Curr Cancer Drug Targets 2003;3:377-383. 